\title{
Growth and physiological responses of Asplenium nidus to water stress
}

\begin{abstract}
Pot experiment conducted in the nursery of Faculty of Forestry, University Putra Malaysia during the periods from May to August, 20006 to investigate the effect of water stress on Asplenium nidus growth performance, transpiration rate and chlorophyll content in fronds. The plants were subjected to five water stress levels; control-watered every day, T1-watered every three days, T2-watered every seven days, T3-watered every fourteen days and T4suspended without watering. Growth parameters were measured every week for a period of sixteen weeks. At the end of experiment, transpiration rate and chlorophyll content were measured as well as dry matter of plants. The results showed that levels of watering did not significantly affect all growth and physiological parameters of Asplenium nidus except T4 which decreased all growth parameter and some physiological responses. T1 had the highest dry matter production. The plants allocated more growth to the shoot under optimum conditions but more growth to the root under stress conditions. This plant was able to adapt and survive without watering for 16 weeks and demonstrate that it can survive in water limited condition.
\end{abstract}

Keyword: Environmental stress, Fern, Forest canopy, Growth response 